\title{
Morphological evaluation of cartilage development in the distal femoral epiphysis of human fetuses
}

\author{
CAL ROSA, J. A.* and LIBERTI, E. A. \\ Anatomy Laboratory, Universidade de São Paulo - USP, Av. Lineu Prestes, 2415, Cidade Universitária, \\ CEP 05508-900, São Paulo, SP, Brazil \\ *E-mail: josyacal@hotmail.com
}

\begin{abstract}
Introduction: The epiphysis cartilage in the knee joint has been widely studied in recent decades due to the joint complexity and its clinical importance. However, there is little information about morphological and ultrastructural aspects of normal cartilage development of the knee joint, particularly in human fetuses of different age, which could contribute to a better understanding of diseases and malformations during development. Materials and Methods: This research was performed with 20 human fetuses covering the age range 16 to 31 weeks of intrauterine life, which were divided into 4 groups $(n=5)$. Morphometric analysis of the distal femoral epiphysis cartilage were realized for the parameters: chondrocytes area, percent density of chondrocyte and extracellular matrix (ECM), as well as qualitative analysis of the distribution, organization and characterization of collagen fibers making use of Picrosirius staining under analysis with polarized light. Results: It was verified that the chondrocytes area and density of ECM increased with advancing age, while the density of chondrocytes decreased. Also in the epiphyseal region, immature cartilage of younger fetuses, initially with characteristics of a proliferation zone starts to present, in older age fetuses, characteristics of a maturation zone, with larger chondrocytes and more distant from each other. In the epiphysis surface the organization and orientation of the collagen fibers changed in different groups, from an irregular orientation in the younger fetuses group, to a pantograph organization in the most developed fetuses group. Conclusion: During fetal development, the maturation of cartilage combines increasing ECM percent density and changes in the collagen fibers organization and orientation, contributing to the required cartilage properties for each stage of its development.
\end{abstract}

Keywords: articular cartilage, femur, fetuses, collagen.

\section{Introduction}

The intense interest and attention given to the knee joint development can be seen from the perspective of the joint complexity and its clinical importance, for example, the growing search for effective therapies for cartilage repair (ARCHER, DOWTHWAITE and FRANCIS-WEST, 2003; ZUSCIK, HILTON, ZHANG et al., 2008) and advances in tissue engineering (HILLEL, TAUBE, CORNISH et al., 2009).

According to Fukazawa, Hatta, Uchio et al. (2009), the description and morphological study of the normal joint components development is required for a better understanding of abnormal morphology, possible defects during the structure development and can serve as a theoretical basis for cartilage repair strategies (CLUZEL, BLOND, FONTAINE et al., 2013).

In addition, the characterization of chondrocytes density and organization in cartilage in the context of growth and maturation would be a step toward understanding the role of such features in governing growth dynamics at the tissue level (JADIN, WONG, BAE et al., 2005).

The purpose of this study is to evaluate the morphological aspects of cartilage development in the distal femoral epiphysis of human fetuses, from the 16 th to the 31 th week of intrauterine life, in order to verify correlation between morphological changes in the distal femoral epiphysis cartilage and fetal development within different age groups.

\section{Materials and Methods}

Forming the experimental groups, it was used twenty (20) human fetuses aged between 16 and 31 weeks, belonging to the didactic collection biorepository of the Department of Biomedical Sciences Institute of Anatomy, University of São
Paulo (ICB III - USP) previously fixed in 10\% formalin and preserved in glycerin, which were divided into four (04) groups $(\mathrm{n}=5)$, as follows: group 1 (Gl): 16-19 weeks, Group 2 (G2): 20-23 weeks, Group 3 (G3): 24-27 weeks and Group 4 (G4): 28-31 weeks.

In all fetuses, the knee region was carefully bilaterally dissected and then decalcified by immersing in ethylenediaminetetraacetic acid (EDTA) at 7\%. Serial sections of 5 micrometers thick were stained by hematoxylin and eosin (HE) for determining the

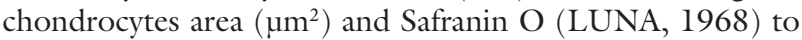
evaluate the density of chondrocytes and extracellular matrix (ECM) in the middle region of the distal femoral epiphysis. For the collagen fibers evaluation, it was used picrosirius staining, analyzed under polarized light (JUNQUEIRA, BIGNOLAS and BRENTANI, 1979).

The data were evaluated in an image system (Carl Zeiss MicroImaging, Axioscope 40 model) and Axiocam camera, connected to the quantification software Axiovision Rel. 4.8 (Zeiss).

The data were statistically compared using the Kruskal-Wallis test (KRUSKAL and WALLIS, 1952) between different groups.

\section{Results and Discussion}

The quantitative results obtained for the chondrocytes area, percent density of chondrocyte and ECM parameters presented significant difference between the groups $(\mathrm{p}<0.05)$ and are shown in Table 1.

Comparing the age, it can be observed increasing values for chondrocytes area. This correlation between the fetuses 
Table 1. Quantitative results for the chondrocytes area, percent density of chondrocyte and ECM. M+SD of chondrocytes area, percent density of chondrocyte and ECM for the groups G1, G2, G3 and G4.

\begin{tabular}{lcccc}
\multicolumn{1}{c}{ Parameters } & G1 & G2 & G3 & G4 \\
\hline Chondrocytes area $(\mu \mathrm{m} 2)$ & $60.7 \pm 1.3$ & $74.8 \pm 9.0$ & $120.0 \pm 9.0$ & $181.8 \pm 63.2$ \\
Percent density of chodrocytes (\%) & $47.5 \pm 9.2$ & $46.7 \pm 6.0$ & $30.1 \pm 4.3$ & $25.1 \pm 2.2$ \\
Percent density of ECM (\%) & $52.7 \pm 9.2$ & $53.2 \pm 6.0$ & $69.8 \pm 4.3$ & $74.8 \pm 2.2$ \\
\hline
\end{tabular}

Statistical significance $\mathrm{p}<0.05$.

development and increasing chondrocytes area can also be verified through the frequency distribution analysis, as shown in the graphic of Figure 1. It can be observed that $84 \%$ of the Gl chondrocytes and $62 \%$ of G2 chondrocytes have areas smaller than $80 \mu \mathrm{m}^{2}$, on the other hand, $85 \%$ of the G3 chondrocytes and $95 \%$ of G4 chondrocytes have areas greater than $80 \mathrm{\mu m}^{2}$.

According to Chen, Johnson, Haudenschild et al. (1995), during endochondral bone formation, chondrocytes in the cartilaginous tissue of long bones progress through a spatially and temporally regulated differentiation program before being replaced by bone. Regarding the size of the chondrocytes, in the initial stage of differentiation, chondrocytes were smaller compared with the following stages. In the second stage, called maturation, chondrocytes had intermediate size, while in the third stage of hypertrophy, those chondrocytes had grown dramatically.

The statistical results for the percent density chondrocyte and ECM parameters presented significant difference between the groups and it was clearly verified that percent density of chondrocytes decreases with advancing age and, on the other hand, the percent density of ECM increases, as shown in the Figure 2 .

Thus, the cartilage tissue maturation during fetal development is related to the changes in the organization of this tissue, where de chondrocytes became disperse. Similar observations were found by Chen, Johnson, Haudenschild et al. (1995) and Jadin, Wong, Bae et al. (2005).

Using picrosirius staining examined under polarized light, it was possible to observe the features that allow the differentiation of each group regarding organization, orientation and predominant type of collagen fibers in the epiphysis surface.

Therefore, Gl has the predominance of type I thick fibers (stained in red, orange and yellow colors) and irregular grid orientation, type I fibers exhibited a transverse orientation pattern, arranged in a compact form in G2. The G3 fetuses was characterized by displaying a lot of type III fibers (stained in green) together with type I thin fibers forming an irregular aspect mesh. In older fetuses (G4), the type I thick fibers were predominant, organized in a pantograph mesh (Figure 3a-d). According to Archer, Dowthwaite and Francis-West (2003), one of the intriguing aspects of the development and maturation of articular cartilage is the collagen orientation changes from an essentially isotropic immature formation, generally oriented parallel to the articular surface, to a non-isotropic arrangement. Williamson, Chen, Masuda et al. (2003) has presented the hypothesis that the tensile biomechanical properties of the articular cartilage surface change during tissue development and these functional changes are related to changes in the collagen network arrangement.

At the middle region of the femoral cartilage, the prevalence was of type II collagen fibers (bluish or greenish) in all groups. The main difference was in Gl, where it was detected the

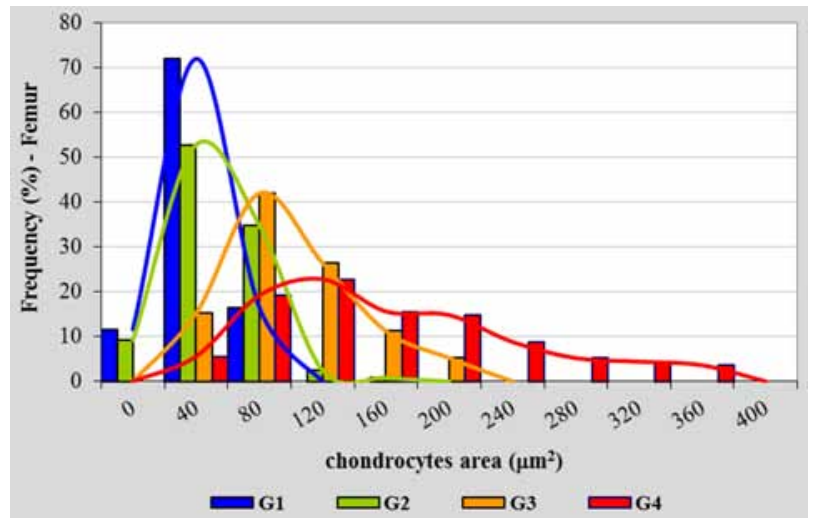

Figure 1. Frequency distribution of the chondrocytes area for the groups G1, G2, G3 and G4. Frequency distribution of the cartilage chondrocytes area. Values of Gl group concentrated between $0-120 \mu \mathrm{m}^{2}$, G2 group concentrated between 0-160 $\mathrm{m}^{2}$, G3 group concentrated between $40-240 \mu \mathrm{m}^{2}$ and G4 group concentrated between $40-400 \mu^{2}$.

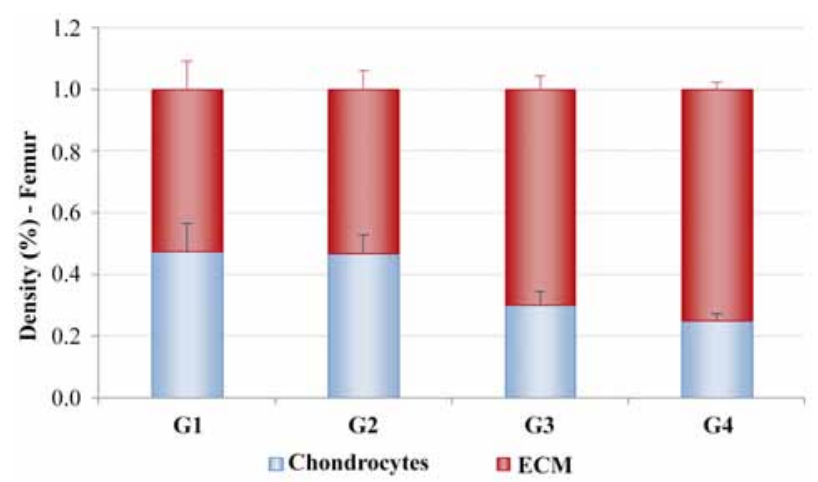

Figure 2. Percent density of chondrocytes and ECM, for the groups G1, G2, G3 and G4. Percent density of chondrocytes and extracellular matrix (ECM), for the groups G1, G2, G3 and G4. Percent density of chondrocytes in blue color. Percent density of ECM in red color. Statistical significance $\mathrm{p}<0.05$.

presence of type I fibers intermingled with of type II fibers (Figure $3 \mathrm{e}-\mathrm{h}$ ).

Regarding the type of collagen fiber found in the epiphysis surface region, Treilleux, Mallein-Gerin, Guellec et al. (1992), studying the location of type I, II and III collagen during the articular cartilage development, observed that types I and II collagen are present in the developing cartilage of the epiphysis, similarly to the findings of this research. 

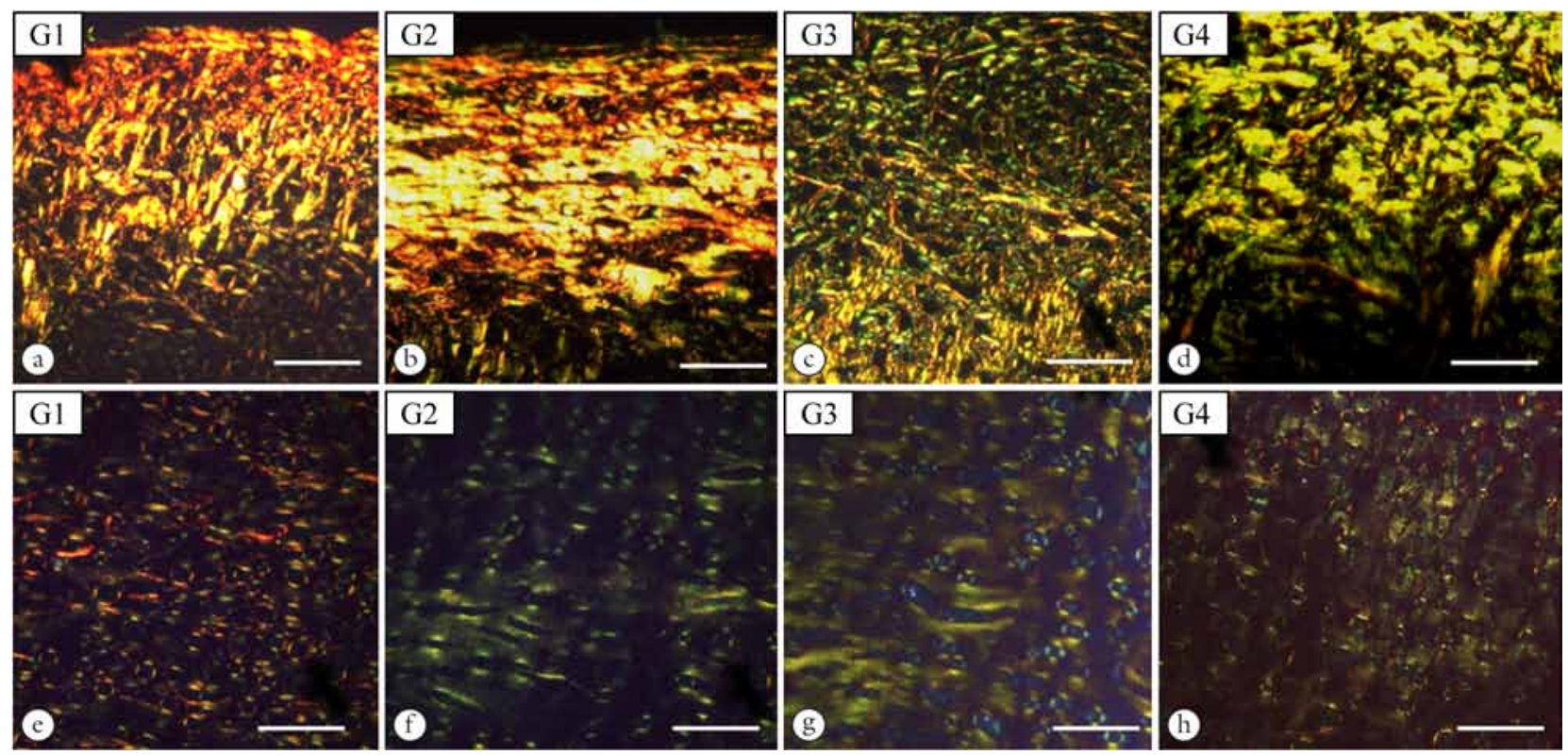

Figure 3. Surface and middle zone of the femoral epiphysis cartilage, in different groups. Surface of the epiphysis cartilage (a-d) and the middle zone of the epiphysis cartilage (e-h), for the groups G1, G2, G3 and G4. Histological technique: Picrosirius analyzed under polarized light. Calibration bar: 50 micrometers.

\section{Conclusions}

In the middle region of the distal femoral epiphysis cartilage, the chondrocytes area increased with advancing age, at the same time, the percent density of chondrocytes decreased and the percent density of ECM increased. The immature cartilage of younger fetuses, initially with characteristics of a proliferation zone, starts to present characteristics of a maturation zone in older age fetuses, with larger chondrocytes and more distant from each other. It was noted that, the cartilage tissue differentiation, during development of this region, is related to the preceding stages of endochondral ossification.

At the epiphysis surface, the collagen fibers organization and orientation changed in the different groups, from an irregular orientation in G1, for a surface parallel orientation in the G2 and finally for a pantograph organization in G4. The type I collagen was predominant in the epiphysis surface in all age groups, contributing to the tensile strength during the cartilage development period, especially in G4, where its fibers was thicker than in the other groups.

During fetal development, the maturation of cartilage combines increasing ECM percent density and changes in the collagen fibers organization and orientation, contributing to the required cartilage properties for each stage of its development, such as tensile strength, resistance to compression and the ability to distribute loads.

Acknowledgements: We would like to thank Ms. RN Prisco (statistical analysis) and Ms. M Righetti (histology preparations).

\section{References}

ARCHER, CW., DOWTHWAITE, GP. and FRANCIS-WEST, PF. Development of synovial joints. Birth Defects Research, 2003, vol. 69, n. 2, p. 144-155. http://dx.doi.org/10.1002/bdrc.10015. PMid:12955858.
CHEN, Q., JOHNSON, DM., HAUDENSCHILD, DR. and GOETINCK, PF. Progression and recapitulation of the chondrocyte differentiation program: cartilage matrix protein is a marker for cartilage maturation. Developmental Biology, 1995, vol. 172, n. 1, p. 293-306. http://dx.doi.org/10.1006/dbio.1995.0024. PMid:7589809.

CLUZEL, C., BLOND, L., FONTAINE, P., OLIVE, J. and LAVERTY, S. Foetal and postnatal equine articular cartilage development: magnetic resonance imaging and polarised light microscopy. European Cells \& Materials, 2013, vol. 26, p. 33-47. PMid:23934790.

FUKAZAWA, I., HATTA, T., UCHIO, Y. and OTANI, H. Development of the meniscus of the knee joint in human fetuses. Congenital Anomalies, 2009, vol. 49, n. 1, p. 27-32. http://dx.doi. org/10.1111/j.1741-4520.2008.00216.x. PMid:19243414.

HILLEL, AT., TAUBE, JM., CORNISH, TC., SHARMA, B., HALUSHKA, M., MCCARTHY, EF., HUTCHINS, GM. and ELISSEEFF, JH. Characterization of human mesenchymal stem cell-engineered cartilage: analysis of its ultrastructure, cell density and chondrocyte phenotype compared to native adult and fetal cartilage. Cells, Tissues, Organs, 2009, vol. 191, n. 1, p. 12-20. http://dx.doi. org/10.1159/000225985. PMid:19546516.

JADIN, KD., WONG, BJ., BAE, WC., LI, KW., WILLIAMSON, AK., SCHUMACHER, BL., PRICE, JH. and SAH, RL. Depthvarying density and organization of chondrocytes in immature and mature bovine articular cartilage assessed by $3 \mathrm{D}$ imaging and analysis. The Journal of Histochemistry and Cytochemistry, 2005, vol. 53, n. 9, p. 1109-1119. http://dx.doi.org/10.1369/jhc.4A6511.2005. PMid:15879579

JUNQUEIRA, LCU., BIGNOLAS, G. and BRENTANI, R. Picrosirius staining plus polarization microscopy: a specific method for collagen detectionin tissues sections. The Histochemical journal, 1979, vol. 11, n. 4, p. 447-455. http://dx.doi.org/10.1007/BF01002772. PMid:91593.

KRUSKAL, WH. and WALLIS, WA. Use of ranks in one-criterion variance analysis. Journal of the American Statistical Association, 1952, vol. 47 , n. 260 , p. 583-621. http://dx.doi.org/10.1080/016214 59.1952 .10483441 
CAL ROSA, J. A. and LIBERTI, E. A.

LUNA, LG. Histologic staining methods of the armed forces institute of pathology. 3rd ed. New York: Mc Graw-Hill, 1968. 62 p.

TREILlEUX, I., MALLEIN-GERIN, F., GUELLEC, D. and HERBAGE, D. Localization of the expression of type I II III collagen, and aggregan core protein genes in developing human articular cartilage. Matrix (Stuttgart, Germany), 1992, vol. 12, n. 3, p. 221-232. http:// dx.doi.org/10.1016/S0934-8832(11)80065-X. PMid:1406455.

WILLIAMSON, AK., CHEN, AC., MASUDA, K., THONAR, EJ. and SAH, RL. Tensile mechanical properties of bovine articular cartilage: variations with growth and relationships to collagen network components. Journal of Orthopaedic Research, 2003, vol. 21, n. 5, p. 872-880. http://dx.doi.org/10.1016/S0736-0266(03)00030-5. PMid:12919876.

ZUSCIK, MJ., HILTON, MJ., ZHANG, X., CHEN, D. and O'KEEFE, RJ. Regulation of chondrogenesis and chondrocyte differentiation by stress. The Journal of Clinical Investigation, 2008, vol. 118, n. 2, p. 429-438. http://dx.doi.org/10.1172/JCI34174. PMid:18246193.

Received January 28, 2015

Accepted October 20, 2015 